\title{
Observation of the stray field of thin film magnetic tips using electron holography
}

\author{
E. Lunedei ${ }^{\text {a }}$, G. Matteucci ${ }^{\text {a, }}$, B.G. Frost ${ }^{\text {b }}$, J. Greve ${ }^{\text {c }}$ \\ ${ }^{a}$ Department of Physics and Istituto Nazionale per la Fisica della Materia, University of Bologna, V/le B. Pichat 6, 40126 Bologna, Italy \\ ${ }^{\mathrm{b}}$ High Temperature Materials Laboratory, Oak Ridge National Laboratory, P.O. Box 2008, Oak Ridge, TN 37831, USA \\ 'Applied Optics Group, Department of Physics, University of Twente, P.O. Box 217, 7500 AE Enschede, The Netherlands
}

\begin{abstract}
The stray field around thin film ferromagnetic tips employed for magnetic force microscopy has been revealed using electron holography. The experimental phase difference maps are in good agreement with simulations. Quantitative flux measurements of the leakage field are obtained.
\end{abstract}

Keywords: Magnetic probes; Leakage fields; Electron hololgraphy

Magnetic Force Microscopy (MFM) is one specific technique, widely used in material science, to determine stray magnetic microfields above the surface of a variety of samples of interest in micromagnetism [1]. In this form of microscopy a sample is raster-scanned by a probe and the physical interaction is monitored. The magnetic field distribution around the tip apex has to be known exactly in order to be able to interpret the acquired image with respect to quantitative magnetization determination.

Electron holography was used to reveal the magnetic field around the tip apex [2]. This technique works through a two-step procedure. The amplitude and phase distributions of the object wave crossing the near apex region of the magnetic tip are first recorded in an electron hologram by superimposing this wave on a reference one. The reconstruction of the object wave is accomplished by optical and/or digital means. As a final result, the holographic method allows the direct display of the stray field around the tip apex together with its quantitative flux measurement which affects the sample when the tip is used in a magnetic force microscope $[2,3]$.

The magnetic probe under investigation is based on a silicon-nitride tip positioned at the end of a cantilever, Fig. 1. The pyramidal tip is vacuum-coated with a $100 \mathrm{~nm}$ of $\mathrm{Co}_{80} \mathrm{Ni}_{20}$. As sketched in Fig. 1, the magnetic material M covers only one of the four tip faces. This probe is then poled in the homogeneous part of a $1 \mathrm{~T}$ field oriented

\footnotetext{
" Corresponding author. Fax: +39-51-630-5153; email: matteucci@gpxbof.df.unibo.it.
}

along the tip axis. A Hitachi HF-2000 field emission electron microscope, operating at $200 \mathrm{kV}$ with a Möllenstedt-type biprism inserted between the first and second intermediate lenses was used to investigate the probe. The objective lens was switched off to exclude any interaction between the magnetic lens field and the sample.

During the electron hologram recording, because of the electron beam (EB) irradiation, the upper surface of the tip acquires an electric charge $E$, Fig. 1 . Therefore, the electron wave travelling in the near apex region is phase modulated by the combined effects of the electric and magnetic stray fields.

Analytical models have been developed to account for both contributions. The electron phase shift due to the

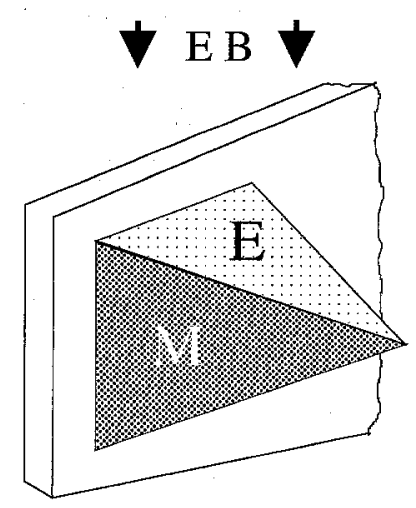

Fig. 1. Sketch of the cantilever and the pyramidal tip. $M$ is the magnetic film, $E$ is the electric charged side. 


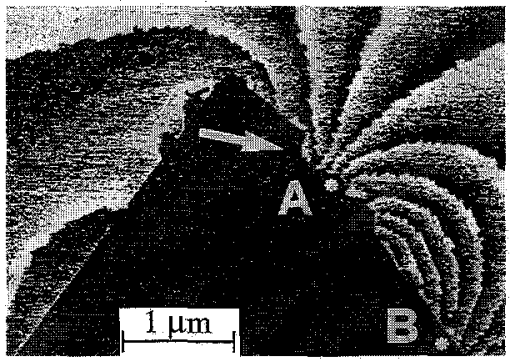

(a)

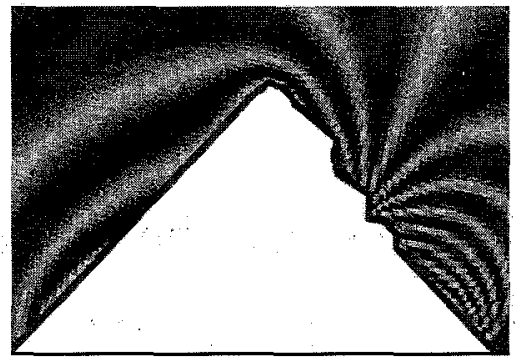

(b)

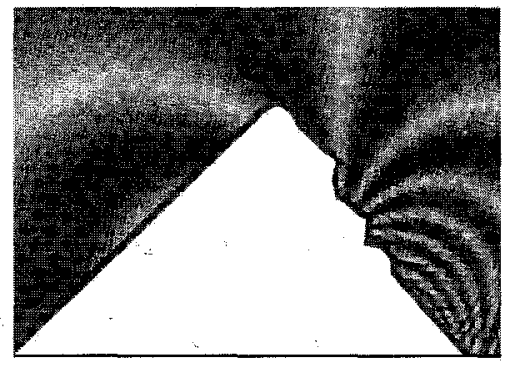

(c)

Fig. 2. (a) Experimental phase map. (b) Simulation of (a). (c) Magnetic lines of force around the tip.

electric potential distribution [4], can be calculated by considering a suitable arrangement of charges on the upper face irradiated by electrons.

The magnetic field leaking from this probe has been simulated with ad hoc developed programs. The magnetic domain structure of the layer is replaced by a uniform distribution of randomly oriented magnetic dipoles. Such a configuration is made to evolve to minimize the total free energy of the magnetic foil. Two main terms were taken into account for energy balance [5], that of dipole-dipole interaction and the energy associated to the interaction between the magnetic moment of each single dipole and the externally applied magnetic field. The limit angular configuration of the film is such that each dipole is oriented parallel to the vectorial sum of the external applied magnetic field and the global magnetic field generated by all other dipoles. Each dipole can rotate freely around the polar angles, but no dipole shift is allowed. This magnetic system evolves from a completely random angular distribution of the dipoles by adjusting their angles until they reach a local minimum energy condition. By repeating this process many times and tabulating the angular configurations reached by the system, we can know the final arrangements that are more likely to be obtained.

In order to map the leakage field around the tip, the phase shift on the electron wave is calculated according to the Aharonov Bohm effect and by applying the Stokes theorem $[2,3]$.

Fig. 2a shows the experimental phase map due to the superposition of electric and magnetic leakage fields around the projected tip image. The magnetic layer deposited on the tip face, parallel to the electron beam (perpendicular to the picture plane) is localized between the white dots $\mathrm{A}$ and $\mathrm{B}$. A strong roughness on the tip surface, covered with the magnetic material, is evidenced by the arrow. In that region, a break on the thin magnetic film is present so that the stray magnetic field is due to the layer between $\mathrm{A}$ and
B. No magnetic material is deposited on the tip face above the arrowed break. In the tip apex region a few lines, which start from $A$ are displayed while, on the tip face coated with the CoNi film, some lines start from A and bend toward $\mathrm{B}$.

Fig. $2 b$ reports the simulation of Fig. 2a. The satisfactory agreement between the experimental result and the simulated map shows the validity of the proposed models.

Fig. $2 \mathrm{c}$ shows the map of the magnetic leakage field alone around the tip. The field trend is analogous to that obtained by experiments carried out on thin magnetic film tips, with a regular flat face surface, completely covered by a gold layer to avoid any charge effect [6].

An estimate of the total flux affecting the surface of a sample placed at a typical working distance in MFM can be made through the evaluation of the total phase difference in Fig. 2c. This flux is determined by counting the number of lines since between two successive ones an amount $\mathrm{h} / \mathrm{e}$ is enclosed $[2,3]$.

These results show the potentiality of electron holography in the study of the stray field of thin film magnetic probes, even in the case of not well-shaped tips. Further work will be aimed at revealing how the magnetic tip and the sample under investigation may affect each other.

\section{References}

[1] U. Hartmann, Adv. Electron. Electron Phys. 87 (1994) 49.

[2] G. Matteucci, M. Muccini and U. Hartmann, Appl. Phys. Lett. 62 (1993) 1839.

[3] G. Matteucci, M. Muccini and U. Hartmann, 'Phys. Rev. B 50 (1994) 6823.

[4] G. Matteucci, G.F. Missiroli, E. Nichelatti, A. Migliori, M. Vanzi and G. Pozzi, J. Appl. Phys. 69 (1991) 1835.

[5] A.H. Morrish, The Physical Principles of Magnetism (Krieger, Malabar, FL, 1983).

[6] B.G. Frost, N.F. van Hulst, E. Lunedei; G. Matteucci and E. Rikkers, Appl. Phys. Lett. 68 (19.96) 1865). 